\title{
Partner selections in public goods games with constant group size
}

\author{
Te $\mathrm{Wu}^{1}$, Feng Fu ${ }^{1,2}$, and Long Wang ${ }^{1 *}$ \\ ${ }^{1}$ Center for Systems and Control, State Key Laboratory for Turbulence and Complex Systems, \\ College of Engineering, Peking University, Beijing 100871, China \\ ${ }^{2}$ Program for Evolutionary Dynamics, Harvard University, Cambridge, Massachusetts 02138, USA
}

(Dated: May 29, 2018)

\begin{abstract}
Most of previous studies concerning the Public Goods Game assume either participation is unconditional or the number of actual participants in a competitive group changes over time. How the fixed group size, prescribed by social institutions, affects the evolution of cooperation is still unclear. We propose a model where individuals with heterogeneous social ties might well engage in differing numbers of Public Goods Games, yet with each Public Goods Game being constant size during the course of evolution. To do this, we assume that each focal individual unidirectionally selects a constant number of interaction partners from his immediate neighbors with probabilities proportional to the degrees or the reputations of these neighbors, corresponding to degree-based partner selection or reputation-based partner selection, respectively. Because of the stochasticity the group formation is dynamical. In both selection regimes, monotonical dependence of the stationary density of cooperators on the group size was found, the former over the whole range but the latter over a restricted range of the renormalized enhancement factor. Moreover, the reputation-based regime can substantially improve cooperation. To interpret these differences, the microscopic characteristics of individuals are probed. We later extend the degree-based partner selection to general cases where focal individuals have preferences towards their neighbors of varying social ties to form groups. As a comparison, we as well investigate the situation where individuals locating on the degree regular graphs choose their co-players at random. Our results may give some insights into better understanding the widespread teamwork and cooperation in the real world.
\end{abstract}

PACS numbers: 89.75.Hc, 87.23.Kg, 02.50.Le

\section{INTRODUCTION}

As a most popular game, the Prisoner's Dilemma (PD) has been widely employed to characterize and elucidate the cooperation conundrum between individual and group interests through pairwise interactions [1, 2, 3, 4, 5, 6, 7, 8]. In many realistic situations ranging from cellular organisms to hunter business to national negotiations, however, multiple agents instead of two individuals are usually involved. While many researchers treated these $\mathrm{N}$-person problems as a summation of many two-person problems [1, 2, 9, 10, 11, 12], the Public Goods Game (PGG) was proposed as a representative of builtin interactions to investigate the multi-person predicament of cooperation, which can be regarded as a natural extension of Prisoner's Dilemma [13, 14, 15]. In a typical example of the PGG, players belonging to a community of $N$ individuals can adopt one of the feasible actions, say cooperation (C) and defection (D). A cooperator donates an amount of $c$ investment to the common pool whereas a defector nothing. The sum is augmented by an enhancement factor $r$ and then equally distributed among all players irrespective of their contributions [13]. In accordance with the name of the PGG, the parameter $r$ should be constrained to be less than the group size of the PGG but larger than unit (i.e., $1<r<N$ ), suggesting that group of cooperators are better off than group of defectors whereas defectors outperform cooperators in any given mixed group [16]. Despite that the group end up maximizing their payoff if all cooperate, the best strategy for a player is to defect, since every invested unit contribution is discounted as a

*Electronic address: longwang@pku.edu.cn return [17]. Thus, the social dilemma of what is best for egoistic individual and what is best for the group arises. According to both classic and evolutionary game theory, cooperators are doomed under natural selection, which is usually at odds with the observations in the real world. Considerable efforts have been expended to find solutions to this plausible paradox.

A variety of measures, such as punishment $[18,19,20,21$, 22], social diversity and the associated diversity of contribution [16, 23, 24], optional participation [13, 14, 17] and image score effect [25, 26, 27], have been proposed to answer the question how large-scale cooperation can evolve and persist stably. In Ref. [23], the authors investigated the influence of two different patterns of contribution on the cooperators' evolutionary fate in the context of the PGG whenever individuals interact along the heterogeneous social ties, and concluded that cooperation can be enhanced if any act of giving is considered to be cooperative, irrespective of the amount of giving. It should be noted that in this work individuals play PGGs with all those directly connected to them, naturally introducing coercion of participation. Different from this assumption, the autarkic 'loner' was introduced as a third strategy besides cooperation and defection in Refs. [13, 14, 17]. It has shown that this voluntary participation efficiently prevents defectors from spreading within the population through self-adjusting the group size of the PGG, leading to the appearance of the cyclic dominance of Rock-Scissor-Paper type. In most such investigations, nevertheless, the effective group size varies over time as the frequencies of these three strategies oscillate in the population.

In most ubiquitous observed public goods type interactions [28, 29, 30], however, the group formation is not always in this way. Due to the restriction of some social norms and, the fact that each individual has the right to decide whether or 
not to attend an activity [31], the group size can neither be arbitrary number nor be equal to the number of one's neighbors. Instead, individuals are usually divided into equal subgroups to accomplish a public target: of these examples are student dormitory clean, public transportation and predator inspection behavior. On the one hand, although large teams, clubs can function most effectively if their members get well along one another, some individuals are easily tempted to free-ride on the public resources without incurring any cost of contribution [32]. On the other hand, it is difficult to accomplish a public task if too few persons engage in it [33]. Thus, one may ask what the invariable group size of the PGG should be, and how it influences the survivability of cooperators [34, 35, 36].

In this paper we set up a minimal model in which individuals of varying social ties maybe participate different numbers of PGGs while the group size of each typical PGG remains constant during the evolutionary process, in line with most already performed public goods experiments where samples (usually students) were actually divided into groups containing equal rather than heterogeneous members [34]. The Barabási-Albert scale-free networks are adopted to represent interpersonal connections, since most natural and artificial networks share much in common with this type of networks [37]. Focal individuals take into account two regimes to select the group members when playing PGGs: degree-based and reputation-based partner selections. In the partner selection based on degree, a focal individual selects his $g$ neighbors with probabilities concerned with their social ties. For the convenience of discussion, we divide the enhancement factor $r$ by the group size $g+1$ to be the renormalized enhancement factor $\delta$. By virtue of numerical simulations, we found that for small group size, the system transforms from one homogeneous state of defectors, after a sharp transition, into the uniform state of cooperators for increasing $\delta$. For large group size, the curve of cooperation lever versus the quantity $\delta$ sees a 'gentle slope', implying that cooperators and defectors can coexist for a wide spectrum of $\delta$. In the partner selection based on reputation, a focal individual chooses his co-players among his neighbors with probabilities associated with their reputations. Intriguingly, cooperation level as a function of $\delta$ monotonically increases almost parallelly as $\delta$ increases responding to different group sizes. Thus, the interplay of group size and selection regime together orients the evolution and accordingly leading to disparate dynamics of the population. Besides, we extend the degree-based partner selection by equipping focal individuals with biases towards their neighbors of different social ties. As a comparison, we also perform the numerical simulation on degree regular graphs.

The rest of this paper is structured as follows. We make a brief introduction of our model in Section II. Numerical results as well as discussions to these accomplished results are presented in Section III. Concluding remarks are drawn in Section IV.

\section{MODEL}

We consider a system with constant population size $N$. The pairwise connections are specified via a Barabási-Albert network, in which each vertex represents an individual. To construct such a network, we start from a small ring evenly embedded with $m_{0}$ nodes. At each time step, a newly added node links to $m$ existing nodes in the instantaneous network following the preferential attachment scheme, i.e. , the probability of an existing node attracting a link is proportional to its current degree [38]. We repeat this process until $N$ nodes are present in the network. Initially, half proportion of the population are randomly assigned to be cooperators $(\mathrm{C})$ and the remaining defectors (D). Instead of assuming compulsory participation in the PGG [23], each individual (focal individual) picks out a fixed number $(g)$ of his neighbors to join in the public enterprize according to the specified partner selection regime. Due to the heterogeneity of connections, different individuals can potentially involve in diverse numbers of PGGs and, the diversity in the numbers is closely associated with individuals' social ties or reputations. The payoff of a certain individual is accumulated over the sum of all the PGGs centered on his neighbors and himself, respectively. Following common practice, individual obtaining higher payoff are more likely to disseminate his strategy. After each round of the game, each individual $i$ compares his payoff $\left(P_{i}\right)$ with that $\left(P_{j}\right)$ of a randomly chosen neighbor $j$ and switches his strategy $s_{i}$ to $s_{j}$ with a probability $T\left(s_{i} \rightarrow s_{j}\right)=\left(P_{j}-P_{i}\right) / M$ whenever $\mathrm{j}$ fares better (provided the payoff difference is positive), with $M$ ensuring the proper normalization and being given by the maximal possible difference between payoffs of $i$ and $j$. Otherwise, he maintains his present strategy.

As for selection regime, two different patterns are considered here. First, given that individuals of varying numbers of social ties play distinctly different role in real-world communities, thus, whenever playing the PGG, a focal individual has the privilege to determine which neighbors to be picked up. For a specific PGG centered on individual $i$, the probability that each of his neighbors is chosen is given by

$$
Q(j)=\frac{k_{j}^{\beta}}{\sum_{j \in \Omega_{i}} k_{j}^{\beta}}
$$

where $k_{j}$ is the number of neighbors of $i$ 's $j t h$ neighbor and $\Omega_{i}$ the neighborhood set of individual $i$. Apparently, the exponent $\beta$ which we define as the weight of participation, uniquely measures to which extent this partner selection is related to degree. In other words, $\beta=0$ represents that all individuals though with heterogeneous social ties have the same opportunity to be selected, indicating that focal individuals view their neighbors indiscriminately. Highly connected individuals behave actively whenever $\beta$ is positive and otherwise corresponds to the opposite situation. Individual picks up deterministically his most connected neighbor and second most connected and so on when the parameter $\beta$ takes the value of infinity.

Besides, in repeated games, rational individuals can acquire information of their neighbors' performance during the past 
moves, which ineluctably has influence upon individual decision making of either continuing to play with these neighbors or replace them with alternative ones, if they exist, in the future rounds. In order for maximizing one's own self-interest, individual tends to team up with his more collaborative neighbors and interact in the Public Goods Game. We, therefore, conceptualize an individual's times of cooperation in the history as his reputation, known to all his neighbors. Explicitly, $R_{i}(t+1)=R_{i}(t)+\delta_{t}$ where $R_{i}(t)$ is individual $i$ 's reputation at time step $t$. The function $\delta_{t}$ takes value of unit if individual plays cooperation $(\mathrm{C})$ with his partners at time step $t$ and zero if he defects (for details see [26] and references thereof). To ameliorate one's own income, a focal individual is inclined to enter partnership with those who frequently cooperate, which means individuals with larger reputations are more likely to engage in more PGGs. Herein, we assume

$$
S(j)=\frac{R_{j}(t)}{\sum_{k \in \Omega_{i}} R_{k}(t)}
$$

where $S(j)$ has the same definition as $Q(j)$ in the formula (1) aforementioned. Evidently, focal individuals measure the cooperativeness of their neighbors based on their long-term performance other than decisions on one shot. A neighbor defects once because of errors or other uncertainties, will not affect his reputation greatly if he immediately retrieves cooperation in next rounds. But if he frequently defect, focal individual would regard him to be a bad one. Or rather, individuals who defect once should not impose great influence upon themselves but these sticking to defection would certainly suffer from being excluded.

To decouple the effect imposed by the heterogenous social ties of individuals situated on the Barabási-Albert network from the effect arising from selection regime, we also carried on our simulation on degree regular graphs, creating from a ring with the nearest- and next-nearest neighbors [40]. To make a clear comparison, the two types of homogeneous and inhomogeneous networks have equivalent average connectivity.

\section{RESULTS AND DISCUSSION}

In our simulations, a population of $N$ individuals is considered and a Barabási-Albert network is generated to specify the connections between them, along which interactions can occur. Initially, equal percentage of cooperators and defectors are randomly distributed among these $N$ nodes. Individuals spread their strategies under replicate dynamics, meaning that the more fit individuals are more likely to subsist and proliferate their strategies within the population. In view that both individual's social ties and reputation can affect one's social popularity, focal individual tends to select his partners according to one of the two features of his neighbors in our model. The synchronous update is adopted for strategy evolution. We shall investigate how these two different selection regimes separately affect the evolutionary fate of cooperators when varying the interacting group size of the Public Goods Game.
Let us first consider the situation of the weight of participation, $\beta$, being zero, wherein each individual is picked up equiprobably when playing PGGs, independent of whatever their social ties are. The fraction of cooperators who survive at equilibrium state is used to measure the evolution of cooperation. We plot the fraction of these cooperators as a function of the renormalized enhancement factor $\delta$ in Fig. 1. Obviously, as the PGG group size contracts, the threshold ( $\left.\delta_{\text {threshold }}\right)$ required above which defectors are unable to wipe out cooperators anymore appreciates. For a given $\delta(<0.87)$, increasing the group size leads to an enhancement of cooperation level. The monotonical dependence becomes vague whenever $\delta$ approaches approximately unit, but cooperation level $(\geq 0.75)$ has been high with respect to the mean value (0.5). As a whole, we can say that large group size readily contributes to the emergence of cooperation in comparison to small ones if it is invariable during the course of evolution. Intuitively, the rationale behind this phenomenon is that increasing group size offers the cooperative individuals with more opportunities to meet more cooperators, thus leading to the formation of cooperative clusters, which can resist replacement of defectors successfully. Consider a cooperator surrounded by three defector and two cooperator neighbors. His expected payoff would be $1.4 \delta-1$ if $g=1$ and $1.8 \delta-1$ if $g=2$. Similarly, defectors can also increase their payoff through exploiting more cooperators per generation as cooperators do for larger group. The benefit resulting from the aggrandized group acts on cooperators and defectors' viability at different rates, the former stronger than the latter, thereby lower quantity of $\delta$ can preferentially select cooperators over defectors, as can be seen in Fig. 1. Interestingly, disparities were found between our findings and the results reported in Ref. [23], in both scenarios with focal individual contributing a fixed amount, $c$, to each PGG but different numbers of possible PGGs he engages in. In our model, the diversity in the group size vanishes. Thus, the heterogeneity in the numbers of the PGGs each individual participates is annealed as compared to the situation of compulsory participation. Notably, this coercion in individual's playing games results in the interactions of individuals as diverse as the population connections. The diversity of fitness deduced from both types of above diversity shrinks in our model, leading essentially to that cooperation level as a function of $\delta$ observes a mild slope compared with results in Ref. [23] for identical conditions with exception of whether or not participation is obligatory. It is noting that the PGG is reduced to the Prisoner's Dilemma for the case of $g=1$, indicating focal individual plus one randomly selected neighbor forms an interacting competed community, the evolutionary equilibrium of the population switches from a uniform state of defectors, after a sharp jump corresponding to a coexistence state between defectors and cooperators, into a homogeneous state of cooperators as $\delta$ increases.

Intriguingly, the monotonous dependence of the cooperative behaviors on the constant group size turns inconspicuous when cooperators greatly dominate the population (see Fig. 1). An inspection of the microscopic property of the evolutionary process is indispensable to understand the appearance of the nontrivial cooperation level versus the group size. 


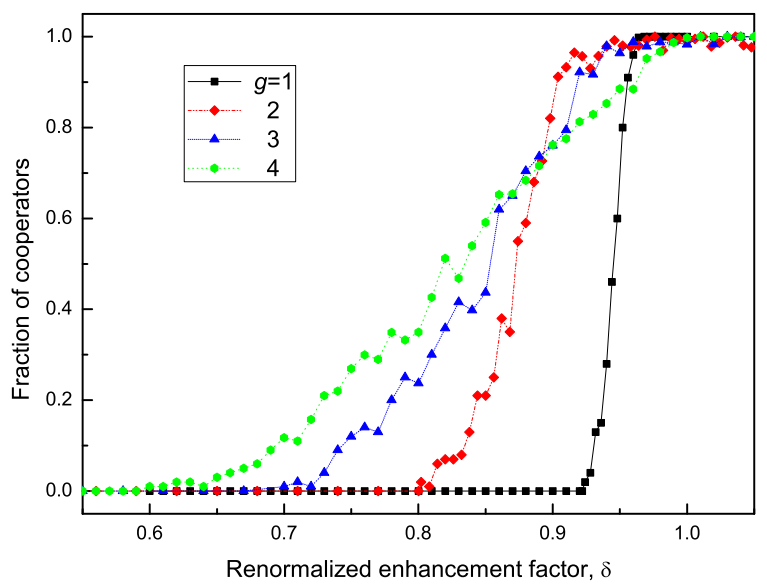

FIG. 1: (Color online) Evolution of cooperation. Fraction of cooperators as a function of the renormalized enhancement factor $\delta$, for different group sizes. Denoting the group size by $g+1$. Population structure is characterized by heterogeneous graph of $N=500$ nodes. We construct the graph following the growth and preference attachment mechanism. Two associated parameters are $m_{0}=4$ and $m=4$. Each data point is averaged over 100 runs, with 10 independent initial strategy distributions in each run.

Fig. 2 shows the cooperator distribution among individuals with different social ties in the population. For $\delta \rightarrow \delta_{\text {threhold }}$ responding to lower cooperation level, the frequency of cooperative strategies declines from high-degree individuals to medium-degree to low-degree ones, consistent with claims made in most previous relevant works [23, 39]. This positive correlation is still existent for medium-value of $\delta$ wherein cooperators and defectors are roughly equally distributed, but grows not so apparent. It is not, however, the same case whenever cooperators dominate defectors whereas unable to homogenize the whole population. The social dilemma in the PGG is as strong as in the Prisoner's Dilemma for small $\delta$. In this settings can cooperators withstand the exploitation of defectors by forming the cluster of compact uniform cooperative community, robust against invasion of egoists through mutual breeding. As $\delta$ rises, the dilemma is gradually lightened. The relaxation is especially evident for $\delta$ approximating 1. Our results approbate this prediction (see Fig. 2). The decisive role hubs play in navigating the evolutionary direction is weakened as opposed to the medium and low-value of $\delta$, and consequently some hubs switch between cooperation and defection from time to time (see the upper panel in Fig. 2).

Unavoidably, rational individuals have capability of memorizing the past performance of their neighbors if the game is repeatedly played. In what follows, we consider another type of selection regime, i.e., reputation-based partner selection. In this regime, focal individuals decide which neighbors to interact with based on the reputations of these neighbors. Fig. 3 demonstrates the associated results. To reach the same nonzero cooperation level, the larger group size, the lower $\delta$ is needed. Interestingly, during the transient phases, cooperation
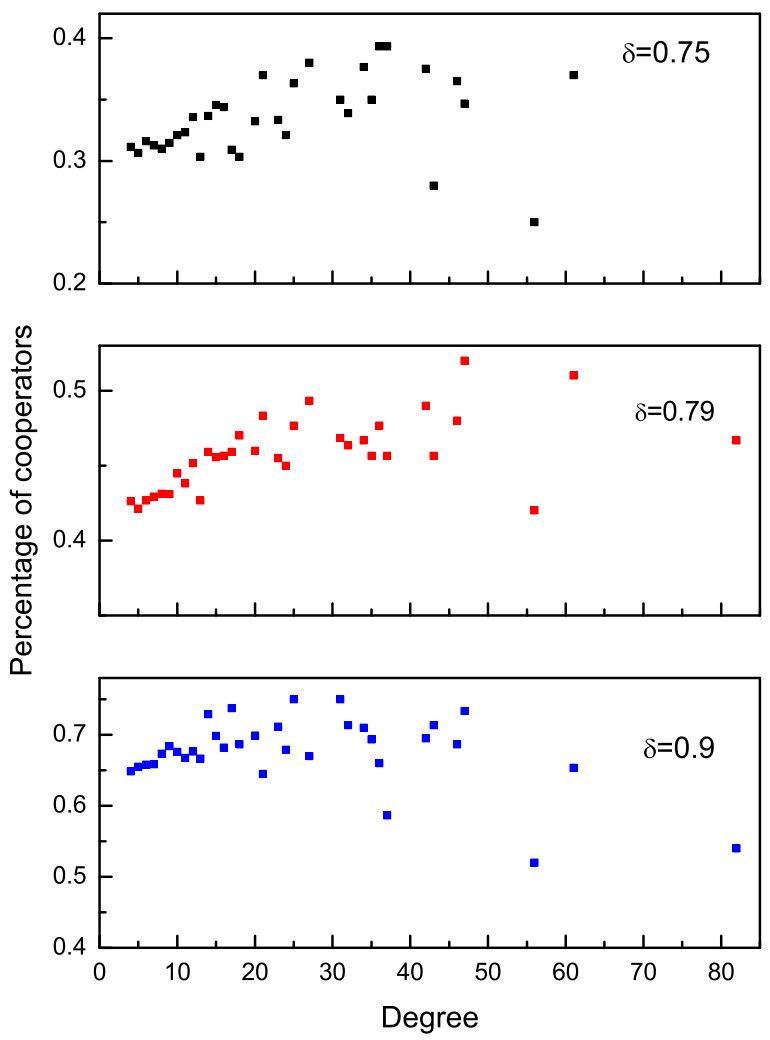

FIG. 2: (Color online) Fraction of cooperators as a function of degree. For a fixed network, each data point is averaged over 300 runs with independent initial strategy distributions. Each run progresses a time of 5000 rounds as transition, and a time window of the next 1000 rounds is intercepted to collect data. A typical value of $g$ is set to be 4 . Cooperation level is $0.27,0.51,0.76$ from the upper panel to the below one, respectively.

level almost parallelly increases with respect to $\delta$ for varying $g$, greatly different from in the case where each individual is selected with equal probability. In the degree-based partner selection, once the social heterogeneous graph describing the population structure is constructed, it would always be static. As a result of the selection just pertaining to individual's social ties, the number of the PGGs each takes part in is unchanged from the perspective of statistics, independent of how often it cooperated in the past. Conversely, a focal individual would alienate his such neighbors with lower reputations by depressing the pick probabilities assigned to them, on the condition he can get access to the local information bearing on the history experience of his neighbors. Individual should be cautious to make his strategy, therefore. For convenience of simulation tractability, each individual was equally initialized with a decimal reputation. If an individual frequently free-rides on the public goods, his reputation progressively becomes compromised relative to mostly cooperative individuals. Apparently, an individual with a low reputation would be ostracized by the communities centered on his neighbors, with an extreme case that an individual of always defecting 


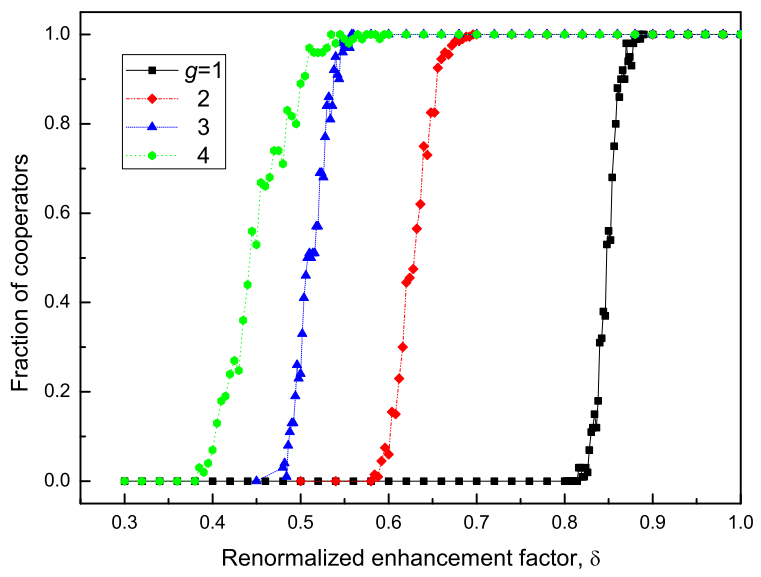

FIG. 3: (Color online) Fraction of cooperators as a function of the parameter $\delta$. Focal individuals each select their group members based on the reputation of their neighbors. Population structure adopted is similar to that in Fig. 1. We have collected the data points as in Fig. 1.

interpolating in a sea of cooperators is destined to die since no individuals are willing to play with him if other alternative neighbors can be found. Herein, this can be thought as a positive feedback correlated the associated members in a group with their reputations. Defectors disrepute themselves as time goes, inducing the repulsion of their neighbors to continue to team with them, which in turn becomes a controlling factor for defectors to be in many PGGs and naturally reduces the opportunities for them to exploit more cooperators. But for cooperators, the inverse holds: focal individuals use the cooperativeness of these cooperators as a choice criterion can help maintain these cooperators a good standing, and thus will attract more neighbors to choose them in future rounds. The exclusion of defectors by their neighbors and the positive assortment among cooperative individuals together brings that configuration fraught with defectors is deteriorating, and will be relegated to cooperate, whereas the ones full of cooperators will reinforce themselves and strengthen their resistance against intrusion of defectors. Thus, impressively lower $\delta$ can induce the emergence and maintenance of cooperation. This reinforcement especially prevents the vacillation of some hubs between cooperation and cooperation as appeared in degreebased partner selection (see Fig. 2 and Fig. 4). Defectors are unable to invade the evolving formed cooperative associates once fully established as the reciprocal altruism of the assortment operates.

We next extend the degree-based partner selection regime to more generous cases by setting the weight of participation, $\beta$, to be a series of discrete values (i.e., $\beta=-1,0,1, \infty$ ). Here $\beta=\infty$ means that a focal individual chooses his most-large and next-most-large neighbors as protagonists if $g=2$, and the like. Results were illustrated in Fig. 5. One can find large $\beta$ favors cooperation for constant group size. This observation can be attributed to the effect of the parameter, $\beta$, on the

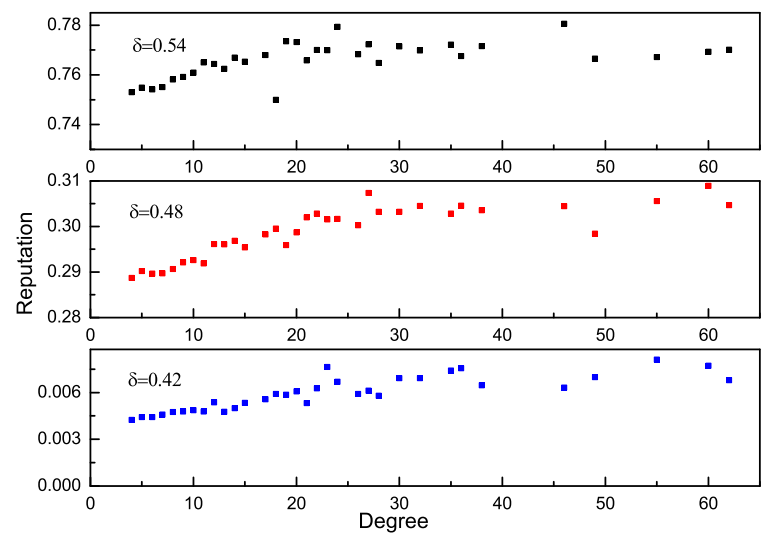

FIG. 4: (Color online) Reputation of individuals as a function of degree at stationary state. A focal individual selects his $g$ co-players among his neighbors based on their reputations. Similar method is adopted to collect data as in Fig. 2. The parameter $g$ is also set to be 4.

heterogeneous numbers of the PGGs each individual participates. Although, the diversity of connections per individual are unchangeable as we adopted a static network of contacts, our assumption of constant group size makes the heterogeneity of network not exactly coincide with that of the numbers of the PGGs each individual actually engages in. Depending on the values of $\beta$, the deviation can be either intensified or weakened. Condition $\beta<0$ indicates individuals are preferential to interact with low-degree ones among their neighbors, which favors unsociable individuals to involve more games while opposes gregarious individuals, who are surrounded with packed neighbors, to experience fewer interactions. On the contrary, value of $\beta$ above zero strengthens the positive correlation between the number of neighbors and the total groups including him, that is, individuals with high-degree and low-degree participate more and fewer PGGs, respectively. The heterogeneity of actual interactions of individuals is partly subject to change of the parameter $\beta$ and the larger the stronger. Thus, large $\beta$ favors the emergence of cooperation. Moreover, the monotonical dependence of degree of cooperation on $\delta$ is quite clear for $\beta$ taking value of the inverse of unit, and ebbs as $\beta$ goes to infinity. This is intuitively straightforward to understand because discrepancy of the two heterogeneity peaks at $\beta=-1$ (among all the explored cases) and wanes for increasing $\beta$. A careful comparison shows that in the degree-based partner selection, the number of the PGGs each individual participates is statistically invariable no matter how his strategy evolves. Unlikely, in another studied selection, one cannot expect how many groups involve a given individual as the decisive role of topology playing in participation is weakened. This hinges in part on the different dynamics of the two selection regimes.

The inherent complexity of games on the scale free graphs makes analytical investigations almost impossible. But, a rough calculation has revealed a conclusion on regular graph, 
which is indeed present but not easily found on scale free graph. Without loss of generality, let us consider the simplest case for regular graphs with degree $k$. Assume that, for the equilibrium local configuration, there have $k_{C}\left(k_{C}^{\prime}\right)$ cooperators and $k_{D}\left(k_{D}^{\prime}\right)$ defectors around a $C(D)$. Because of the updating rule (local competition between nearest neighbors) we used, a cooperator on average has more cooperator neighbors than a defector. Namely, the assortment between cooperators is induced. Hence, we have $k_{C}=k_{C}^{\prime}+\Delta k$ with $\Delta k>0$. In addition, we have the same renormalized enhancement factor $\delta$ for different group sizes.

For group size $g=2$, the expected average payoff of a cooperator and a defector is given by

$$
\begin{gathered}
\bar{f}_{C}=\delta \frac{k_{C}}{k}+\delta-1 \\
\bar{f}_{D}=\delta \frac{k_{C}^{\prime}}{k}
\end{gathered}
$$

Thus the payoff difference for $g=2$ is $\Delta f_{2}=\bar{f}_{C}-\bar{f}_{D}=$ $\delta \frac{\Delta k}{k}+\delta-1$. Analogously, for $g=3$, the expected payoff of a cooperator and a defector can be expressed as

$$
\begin{gathered}
\bar{f}_{C}=\delta\left(\frac{2 k_{C} k_{D}}{k(k-1)}+\frac{2 k_{C}\left(k_{C}-1\right)}{k(k-1)}\right)+\delta-1 \\
\bar{f}_{D}=\delta\left(\frac{k_{C}^{\prime} d_{D}^{\prime}}{k}+\frac{2 k_{C}^{\prime}\left(k_{C}^{\prime}-1\right)}{k(k-1)}\right)
\end{gathered}
$$

Thus the payoff difference for $g=3$ is $\Delta f_{3}=\bar{f}_{C}-\bar{f}_{D}=$ $\delta \frac{2 \Delta k}{k}+\delta-1$. Obviously, we have $\Delta f_{3}>\Delta f_{2}$. This means that larger group size increases the payoff of cooperators more than that of defectors. As a result, larger group size requires a lower critical $\delta$ for the emergence of cooperation, a well consistent analytical prediction for the simulation experiments (see Fig. 6). Moreover, this effect exists in any type of network no matter how heterogeneous it is [10].

Taking together, although the physical networks used to specify the population structure are independent of evolutionary courses, the actual game interactions of focal individuals can be adjusted over time in our minimalist model, thus leading to that the interaction graph for a given individual does not overlap with the learning graph being in concord with the population structure. In Ref. [41], the authors have explored the evolutionary dynamics on graphs with breaking the coincidence of the interaction graph and the replacement graph. The approach of generating the two subgraphs in our model is somewhat different from that in Ref. [41], where difference of the two graphs can be exactly adjusted by tuning a model parameter. In present work, as long as the parameter $\beta$ takes finite value in the degree-based partner selection, focal individuals each can encounter his $g$ neighbors probabilistically in each round. This dynamical constitution of interacting group makes this deviation to be dynamically changed and impossible to be precisely predicted. Then our model can fall somewhere between games on static graphs and coevolution of individual strategy and neighborhood [6, 42, 43] in the sense

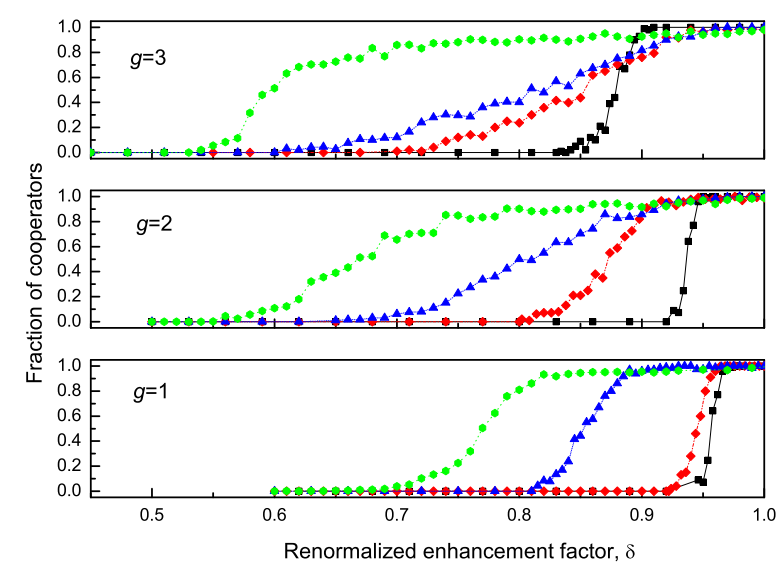

FIG. 5: (Color online) Fraction of cooperators as a function of the parameter, $\delta$, with different values of the participation weight, $\beta$. Lines with squares, diamonds, triangles, circles correspond to the values of $\beta$ being $-1,0,1, \infty$. Population structure adopted is similar to that in Fig. 1. We have collected the data points as in Fig. 1.

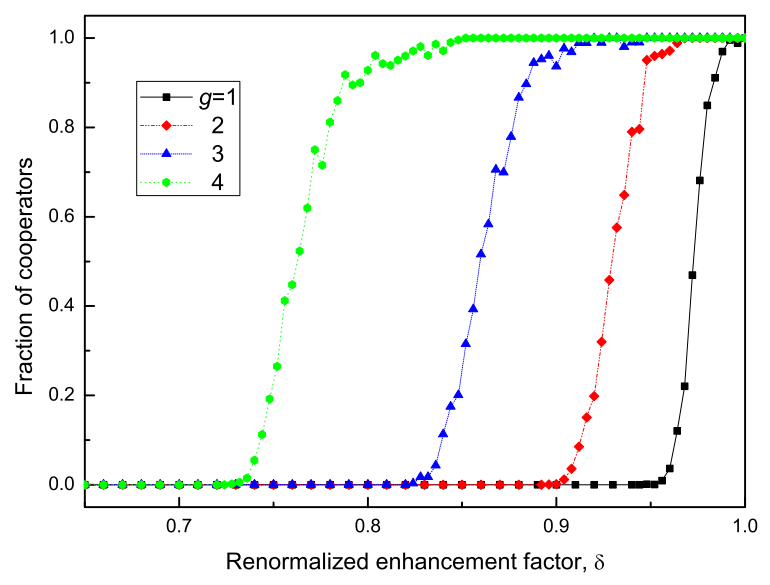

FIG. 6: (Color online) Fraction of cooperators as a function of the parameter, $\delta$, with different group sizes, $g+1$. Population structure is specified by a degree-regular graph, in which each vertex has four neighbors. Focal individuals choose their remaining group members due to the degree-based partner selection as in Fig. 1. Each data point is averaged over 300 runs with independent initial strategy distributions.

that individuals not only update their strategies but also dynamically choose group members from their neighborhood. Noting that focal individuals propose to enter collective actions and have choosiness towards their neighbors of different characteristics. This is clearly an unidirectional selection but simpler than that in Ref. [32] investigated experimentally. The case of random selection (i.e., $\beta=0$ ) eliminates the preferences of focal individuals towards neighbors, but imposes a great influence on the evolution of cooperative behavior just 
similar to a gradual but long lasting process of erosion by water to the formation of deep valleys. Extension of the random selection (i.e., non-zero values of $\beta$ ) closely reflects such reallife situations where individuals have different preferences towards individuals with diverse social ties.

In the case of participation being compulsory, an increase in the average group size is detrimental to the survival of cooperation, holding for both two investment schemes adopted (see [23] for details). Conversely, our findings show that increase in the average group size (i.e., $g+1$ ) is beneficial to the buildup of cooperation on heterogeneous structured population. In Ref. [23], increase of the average connectivity leads to increase in the connectedness of the graph, a disadvantageous feature against the establishment of cooperation. In our model, since we have fixed the average degree of the graph, the heterogeneity of the population is unchanged. Besides, we have normalized the enhancement factor $r$ divided by $g+1$ as $\delta$, the overall scaling of the value of $r$ deduced from increase in group size is automatically incorporated. Remaining differences between curves (see Fig. 1) should be attributed to other factors. For small fixed group size, any individual can choose $g$ group members from his more than $g$ neighbors. This flexibility of selection, which softens up the heterogeneity of actual interacting network, is lessened as $g$ increases. Of an extreme $g$ being set to be 4 , individuals accounting for a large proportion of the population have no alternative neighbors but to confront all their neighbors, meaning that the heterogeneity of interacting network is much closer than that of cases of small $g s$, to the heterogeneity of the scale free graph defining the population structure. This can explain the rough monotonical dependence of cooperation level on the group size $g+1$. Similarly, when we alter the participation weight, $\beta$, increase of it also plays a striking positive role in shrinking the divergence of the actual interacting network from the population structure. Thus, for constant group size, cooperation is convenient to emerge and be maintained for large $\beta \mathrm{s}$, consistent with simulation results (see Fig. 5).

In contrast with degree-based partner selection, the population structure is no longer an overriding determinant on who-meets-whom even statistically in reputation-based selection regime. Focal individuals can unidirectionally [32] adjust their preferences towards their neighbors after each round according to their reputations, tantamount to a type of 'soft' punishment where no cost is involved with punishers and the punished. Focal individuals potentially exert punishment on their neighbors of ill repute [44, 45] by excluding them in the future collective behaviors without damaging their own reputations [45]. Thus only those continuously upholding good reputations will not suffer from being excluded and herein acquire more opportunities of help and being helped (i.e., direct reciprocity). This selection regime in effect avoids 'the second-order free-rider problem' because punishers withdraw interactions with the punished at no cost to themselves. Thus, cooperation should emerge for substantially small $\delta$ analogous to degree-based regime, confirmed by the simulation results (see Fig. 1 and Fig. 3). We finally point out that in both selection regimes, irrespective of the social ties or reputations, no individual can be absolutely banished in that each individual would participate at least one PGG centered on himself.

\section{CONCLUSION}

We have proposed a model to study the effect of the constant group size on the evolution of cooperation. The interactions of individuals was metaphorized by the Public Goods Game. Two selection regimes were introduced for a centered individual to pick up a fixed number of players from his neighbors, according to their degrees or reputations, which we refer to as partner selection based on degree or partner selection based on reputation. The centered individual adding these chosen neighbors constitutes an interacting group and plays the PGG. Whenever individuals interact without memory effect (only considering the social viscosity of neighbors), on the one hand large group improves the payoff of both cooperators and defectors, but the former at a larger rate; on the other hand, large group strengthens the heterogeneity of the actual interactions. These two considerations together leads to that large groups favor cooperation more than small ones. In extended cases we found increase of the participation weight plays a principal role in promoting cooperation for a given group size. Later, we investigate how cooperation evolves under the reputation-based selection regime. The positive correlation between individual's reputation and the number of the PGGs per individual forms a positive feedback, which enables centered individuals to reply promptly to frequently defective neighbors. As a consequence, cooperation can be induced to a higher level than in the partner selection based on degree, where individual is inept to displace the members in the community centered on it from the viewpoint of statistics. Besides, we scrutinize the microscopic characteristics to find that, when the selection is progressing based on reputation, the hubs 'loyally' play a leading role in enhancing and stabilizing cooperation in the whole range of the renormalized enhancement factor $\delta$, which is shortened in the degree-based selection regime, offering a better interpretation to the aggregate observations. We also mathematically prove that expansion in the group benefits cooperators more than defectors in a homogeneous population, and this effect can be generalized to any type of network, particularly scale free network. Heterogeneity of the PGGs per individual combining the partner selection regime together leads to rich dynamics on heterogeneous population.

\section{Acknowledgments}

The authors are supported by NSFC (Grant Nos. 60674050, 60736022 and 60528007), National 973 Program (Grant No. 2002CB312200), National 863 Program (Grant No. 2006AA04Z258), and 11-5 project (Grant No. A2120061303). F. F. also gratefully acknowledges the support from China Scholarship Council (2007U01235). 
[1] R. Axelrod, The Evolution of Cooperation (Basic Books, New York, 1984).

[2] R. Axelrod and W. D. Hamilton, Science 211, 1390 (1981).

[3] A. Traulsen, M. A. Nowak, and J. M. Pacheco, Phys. Rev. E 74, 011909 (2006).

[4] J. Vukov, G. Szabó, Phys. Rev. E 71, 036133 (2005).

[5] J. Vukov, G. Szabó, and A. Szolnoki, Phys. Rev. E 73, 067103 (2006).

[6] M. G. Zimmermann and V. M. Eguíluz, Phys. Rev. E 72, 056118 (2005).

[7] Z.-X. Wu, X.-J. Xu, Z.-G. Huang, S.-J Wang, and Y.-H. Wang, Phys. Rev. E 74, 021107 (2006).

[8] J. M. McNamara, Z. Barta and A. I. Houston, Nature 428, 745 (2004).

[9] W. X. Wang, J. Ren, G. R. Chen, B. H. Wang, Phys. Rev. E 74, 056113 (2006).

[10] H. Ohtsuki, C. Hauert, E. Lieberman, M. A. Nowak, Nature 441, 502 (2006).

[11] J. Hofbauer, K. Sigmund, Evolutionary Games and Population Dynamics (Cambridge Univ. Press, Cambridge, UK, 1998).

[12] C. Hauert, M. Doebeli, Nature 428, 643 (2004).

[13] C. Hauert, S. D. Monte, J. Hofbauer, K. Sigmund, Science 296, 1129 (2002).

[14] G. Szabó, C. Hauert, Phys. Rev. Lett. 89, 118101 (2002).

[15] D. Semmann, H. J. Krambeck, M. Milinski, Nature 425, 390 (2003).

[16] M. Doebeli, C. Hauert, T. Killingback, Science 306, 859 (2004).

[17] C. Hauert, S. D. Monte, J. H. Hofbauer and K. Sigmund, J. Theor. Biol 218, 187 (2002).

[18] T. H. Clutton-Brock and G. A. Parker, Nature 373, 209 (1995).

[19] A. Dreber, D. G. Rand, D. Fudenberg and M. A. Nowak, Nature 452, 348 (2008).

[20] C. Hauert, A. Traulsen, H. Brandt, M. A. Nowak, K. Sigmund, Science 316, 1905 (2007).

[21] B. Rockenbach and M. Milinski, Nature 444, 718 (2006).

[22] E. Fehr and S. Cächter, Nature 415, 137 (2002).

[23] F. C. Santos, M. D. Santos, and J. M. Pacheco, Nature 454, 213 (2008).

[24] M. Perc and A. Szolnoki, Phys. Rev. E, 77, 011904 (2008).
[25] H. Brandt and K. Sigmund, Proc. Natl. Acad. Sci. USA 102, 2666 (2005).

[26] F. Fu, C. Hauert, M. A. Nowak, and L. Wang, Phys. Rev. E 78, 026117 (2008).

[27] C. Wedekind and M. Milinski, Science 288, 850 (2000).

[28] K. G. Binmore, Playing Fair: Game Theory and the Socail contract (MIT Press, Cambridge, 1994).

[29] L. A. Dugatkin, Cooperation among Animals: An Evolutionary Perspective (Oxford Universtiy Press, Princeton, 1995).

[30] A. M. Colman, Game Theory and Its applications in the Socail and Biological Sciences (Butterworth-Heinemann, Oxford, 1995).

[31] K. H. Lee, C.-H. Chan, P. M. Hui, D. F. Zheng, Physica. A 387, 5602 (2008).

[32] G. Coricelli, D. Fehr, G. Fellner, Journal of Conflict Resolution 48, 356 (2004).

[33] M. Haag, R. Lagunoff, J. Eco. Theor 135, 68 (2007).

[34] M. Milinski, D. Semmann, H. J. Krambeck, and J. Marotzke, Proc. Natl. Acad. Sci. USA 103, 3994 (2006).

[35] M. Milinski, R. D. Sommerfeld, H. J. Krambeck, F. A. Reed, and J. Marotzke, Proc. Natl. Acad. Sci. USA 105, 2291 (2008).

[36] C. B. Froyn, J. Hovi, Eco. Lett. 99, 317 (2008).

[37] L. A. N. Amaral, A. Scala, M. Barthélémy, and H. E. Stanley, Proc. Natl. Acad. Sci. USA 97, 11149 (2000).

[38] A. L. Barabási, R. Albert, Science 286, 509 (1999).

[39] Z.-H. R, X. L, and X.-F. W, Phys. Rev. E 76, 027101 (2007).

[40] G. Szabó, G. Fáth, Evolutionary games on graphs, Phys.Rep, 446, 97(2007).

[41] H. Ohtsuki, M. A. Nowak, and J. M. Pacheco, Phys. Rev. Lett. 98, 108106 (2007).

[42] M. G. Zimmermann, V. M. Eguíluz, and M. S. Miguel, Phys. Rev. E 69, 065102 (2004).

[43] J. M. Pacheco, A. Traulsen, and M. A. Nowak, Phys. Rev. Lett. 97, 258103 (2006).

[44] E. Fehr, Nature 432, 449 (2004).

[45] B. Rockenbach and M. Milinski, Nature 457, 39 (2009).

[46] J. M. McNamara, Z. Barta, L. Fromhage and A. I. Houston, Nature 451, 189 (2008). 